\title{
El sistema ANDON, como herramienta fundamental para disminuir el tiempo de respuesta y eliminar los defectos en línea de panel
}

\section{The ANDON system, as a fundamental tool to decrease the response time and eliminate defects in the panel line}

\author{
MARTÍNEZ-HERNÁNDEZ, Julio Cesar†*, CRUZ-SOLÍS, Edgar Jesús, HERNÁNDEZ-LUNA, Aldo \\ y HERNÁNDEZ-HILARIO, Raymundo
}

Instituto Tecnológico Superior de Huauchinango, México.

ID $1^{\text {er }}$ Autor: Julio Cesar, Martínez-Hernández / ORC ID: 0000-0001-9528-156X, Researcher ID Thomson: I-3507-2018 arXiv Author ID: 0000-0001-9528-156X, CVU CONACYT ID: 904537 ID $1^{\text {er }}$ Coautor: Edgar Jesús, Cruz-Solís / ORC ID: 0000-0003-4083-0888, Researcher ID Thomson: I-4334-2018, arXiv
Author ID: 0000-0003-4083-0888, CVU CONACYT ID: 904718

ID $2^{\text {do }}$ Coautor: Aldo, Hernández-Luna / ORC ID: 0000-0002-7717-5314, Researcher ID Thomson: I-1165-2018, CVU CONACYT ID: 441305

ID $3^{\text {er }}$ Coautor: Raymundo, Hernández-Hilario / ORC ID: 0000-0003-2426-2681, CVU CONACYT ID: 1064091

DOI: $10.35429 /$ JIE.2020.12.4.30.41

Recibido Abril 20, 2020; Aceptado Junio 30, 2020

\section{Resumen}

El tiempo es considerado como un elemento fundamental en cualquier tipo de industria, motivo por el cual es necesario optimizarlo, las empresas que no puedan mejorar sus procesos a través de la reducción de tiempos de espera o tiempos muertos, sufren de graves afectaciones en su operación diaria. La empresa en estudio, presenta graves problemas en la línea de panel, por ejemplo, hojas de laminadas, mal corte, derrame de recubrimiento, rompimiento de papel, para la operación de esta línea se cuenta con un tiempo disponible de 48 horas, es importante señalar que solo el $71 \%$ del tiempo es productivo. El objetivo de este estudio es implementar un sistema ANDON, a través de la identificación de problemas que afectan la producción, con el propósito de reducir los tiempos de respuesta en la Línea de Panel. ANDON es un sistema de comunicación visual y auditivo utilizado en la industria que permite a los trabajadores interactuar de manera simple para la solución de problemas que se presenten durante la jornada laboral en el momento en que ocurren (Vera, 2018). Este proyecto contribuye a la mejora continua de la línea de panel, así como un cambio en la filosofía de trabajo de los operadores.

\begin{abstract}
Time is considered a fundamental element in any type of industry, which is why it is necessary to improve it, companies that cannot improve their processes through the reduction of waiting times or downtime, problems of serious damage to their operation daily. The company under study presents serious problems in the panel line, for example, laminated sheets, poor cutting, spillage of coating, paper tear, for the operation of this line there is an available time of 48 hours, it is important note that only $71 \%$ of the time is productive. The objective of this study is to implement an ANDON system, through the identification of problems that affect production, in order to reduce response times on the Panel Line. ANDON is a visual and auditory communication system used in the industry that allows workers to interact in a simple way to solve problems that arise during the workday when they occur (Vera, 2018). This project contributes to the continuous improvement of the panel line, as well as a change in the work philosophy of the operators.
\end{abstract}




\section{Introducción}

Actualmente todas las empresas buscan conservar la calidad del producto final de una manera más segura y económica (Avila, 2015). La empresa en estudio es una de las muchas compañías en México que se encargan en producir embalaje secundario, este depende de un proceso sistemático mediante el cual los productos elaborados son protegidos para su traslado del sitio de producción al sitio de consumo (Velázquez, 2012).

El éxito de diversas empresas no se basa solo en las ventas u ofreciendo precios bajos, sino que depende demasiado de la satisfacción de sus clientes, con respecto a calidad, entrega, flexibilidad y confiabilidad con base en la familia de productos que se les ofrece (Wilches, 2013).

Por otra parte, la empresa debe tener una constante actualización dentro de la familia de productos, esto la mantendrá a la vanguardia dentro del mercado, los japoneses Eiji Toyoda y Taichi Ohno implementaron las herramientas Lean Manufacturing para optimizar y maximizar las oportunidades de mejora presentadas dentro de los procesos (Socconini, 2008).

La empresa en estudio está orientada a la manufactura de empaques de tipo Honeycomb, que es un material hecho de celdas hexagonales recubierto de papel kraft virgen, utilizado por distintas industrias como son: electrodomésticos, alimenticias, automotriz etc., así mismo, cuenta con cinco líneas (Chs, Panel, Convertion, Mabe'S, Cornerboard y Corner Board), el desarrollo del proyecto se enfocó en la línea de panel por su elevada frecuencia de problemas en los procesos que la integran, cabe mencionar que de esta línea depende que el siguiente proceso sea factible.

Para que los empaques tipo Honeycomb cumplan con los estándares de calidad que se manejan en la línea, las máquinas que lo conforman deben de tener un buen desempeño, de tal manera que se cumplan las especificaciones (largo, ancho, grosor, peso, secado y resistencia), sin salir del rango de aceptación.
De acuerdo con los problemas mencionados anteriormente, se realizaron distintos análisis en diferentes subprocesos que integran la línea con la finalidad de conocer las áreas críticas, esta información permite plantear y llevar a cabo medidas correctivas.

De acuerdo con conocimiento específico del proceso productivo en el área de panel. Como alternativa de solución, se propuso la implementación de un sistema ANDON, mismo que, establece una comunicación sencilla, de tal manera que los operadores conozcan que actividades deben realizar durante un problema presentado en la línea, de una manera eficiente, esto ayudara a incrementar los niveles de calidad y productividad con el mínimo costo posible, también evitar que los defectos pasen a la siguiente línea de producción.

ANDON era conocido en la antigüedad por los japoneses como "lámpara", la cual estaba hecha de segmentos de papel colocados alrededor de una base con una vela en su interior y la tapa descubierta, funcionaba como una señal visual que a la distancia daba un mensaje para comunicar algo (Socconini, 2008). Esta herramienta es un elemento del principio Jidoka que, mediante ingeniosos mecanismos, detecta cuando ocurre una falla y entonces, con una señal, avisa al operador que se ha generado un problema.

Con la implementación del presente proyecto de investigación se pretende cumplir las siguientes metas: mejorar la calidad, mejorar el tiempo de respuesta, aumentar la seguridad y mejorar la comunicación. Así mismo, se pretende optimizar el proceso productivo, disminuyendo desperdicios y reprocesos, generando experiencia del trabajo realizado por los operadores, cuando hay alguna falla de las máquinas o en el material.

La implementación del sistema ANDON no es compleja, pero es importante llevarla a cabo para la mejora en la línea, conocidos como señales visuales y auditivas que son colocados estratégicamente en esta misma. Los seres humanos captamos información por medio de nuestros sentidos, el sentido de la vista con el que más captamos, con un $80 \%$, seguido del oído con un $10 \%$, el olfato con $5 \%$, el gusto con $3 \%$ el tacto con 1\% (Socconini, 2008). 
Dentro de las actividades a realizar, se pretende instalar indicaciones visuales y de audio que se identifican y entienden con facilidad, estas señales son eficientes, autorreguladas y las manejan los operadores. Esta información se puede utilizar para identificar, instruir o indicar que existe una condición normal o anormal y que

acción realizar para corregirla.

\section{Metodología}

De acuerdo con Hernández (2010), las investigaciones explicativas son más estructuradas que los estudios con los demás alcances y, de hecho, implican los propósitos de estos (exploración, descripción y correlación o asociación); además de que proporcionan un sentido de entendimiento del fenómeno a que hacen referencia. También está dirigido a responder por las causas de los eventos y fenómenos físicos o sociales. Se enfoca en explicar por qué ocurre un fenómeno y en qué condiciones se manifiesta, o por qué se relacionan dos o más variables.

Con base en la investigación documental realizada, la metodología propuesta se muestra en la figura 1, en ella se describen las etapas desarrolladas para lograr el objetivo del proyecto priorizando las necesidades y problemáticas presentadas en este caso de estudio.

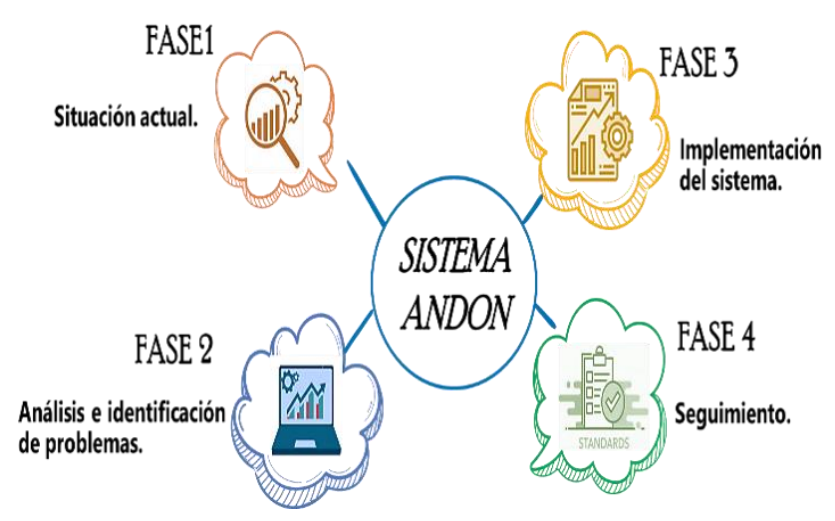

Figura 1 Metodología propuesta Fuente: Elaboración propia

\section{Situación actual}

La línea de panel está integrada por un total de trece maquinas como se muestra en la tabla 1 , es importante que cada máquina trabaje de manera adecuada, ya que de esta depende que el producto final cumpla con los estándares de calidad que maneja la empresa. Así mismo en la figura 2 se muestra el layout del área en estudio.

\begin{tabular}{|l|l|}
\hline \multicolumn{2}{|c|}{ Estaciones de trabajo } \\
\hline 1. Porta rollos & 8. Compresión Deck \\
\hline 2. Michelman & 9. Trim Slitter \\
\hline 3. Core Cart & 10. Trituradoras \\
\hline 4. Rodillo jalador & 11. Slitter sierra \\
\hline 5. Mesa de expansión & 12. Stacker \\
\hline 6. Glue Coater & 13. Emplayadora \\
\hline $\begin{array}{c}\text { 7. Placas de } \\
\text { calentamiento }\end{array}$ & \\
\hline
\end{tabular}

Tabla 1 Procesos que integran la línea de panel Fuente: Elaboración propia

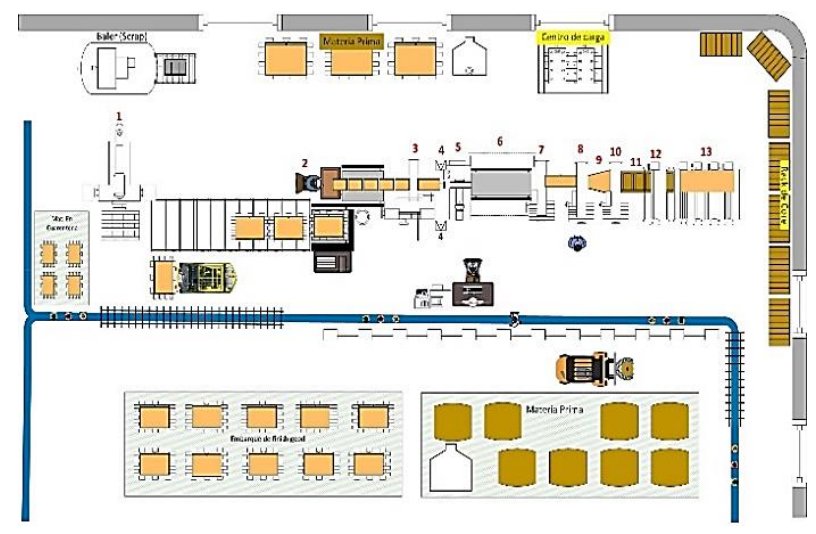

Figura 2 Layout Panel Line Fuente: elaboración propia

Actualmente se cuenta con turnos laborales de 12 horas, en cada turno se encuentran al redor de 25 trabajadores la mayor parte en el área de producción que se dividen en las diferentes áreas, para preparar y ajustar las maquinas se dispone de 20 minutos en promedio, una vez arrancada la línea se presentan diferentes fallas durante el día, por cada problema presente en el día se estima que hay un desperdicio de 15 a 20 piezas como mínimo. La figura 3 muestra la estación de trabajo de Core Cart. 


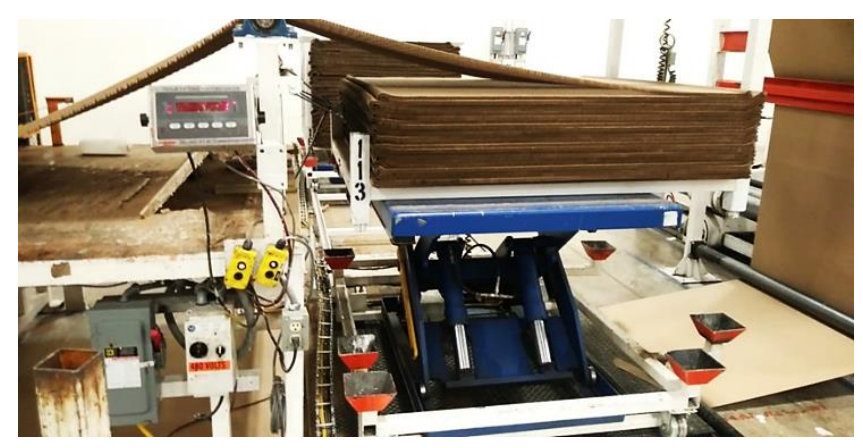

Figura 3 Estación Core Cart

Fuente: Empresa (caso de estudio)

A continuación, se describen algunos de los problemas críticos en las estaciones de trabajo que integran la línea de panel como se muestra en la tabla 2:

\begin{tabular}{|l|l|}
\hline \multicolumn{1}{|c|}{ Estación } & \multicolumn{1}{|c|}{ Problema crítico } \\
\hline Stacker & $\begin{array}{l}\text { El error que se presenta cuando } \\
\text { en la línea el material (core o } \\
\text { papel) se descalibra, en este } \\
\text { tipo de casos el operador no } \\
\text { puede solucionar el problema } \\
\text { sin embargo el indicado es el } \\
\text { líder de la línea, sin embargo, } \\
\text { solo se entera del problema } \\
\text { hasta que el operador se lo } \\
\text { indica. }\end{array}$ \\
\hline Glue Coater & $\begin{array}{l}\text { El problema que presenta se le } \\
\text { conoce como delaminación del } \\
\text { material porque cuando no se } \\
\text { suministra suficiente material } \\
\text { (pegamento) este no aplica lo } \\
\text { necesario y el producto que } \\
\text { sale no obtiene la suficiente } \\
\text { rigidez. }\end{array}$ \\
\hline Triturado & $\begin{array}{l}\text { la falla que se destacó es } \\
\text { originada cuando la maquina } \\
\text { se llega a trabar y si no es } \\
\text { solucionado de manera } \\
\text { inmediata este error ocasiona } \\
\text { que salga material defectuoso, } \\
\text { esto afecta de manera directa el } \\
\text { siguiente proceso (Slitter } \\
\text { sierra). }\end{array}$ \\
\hline
\end{tabular}

Tabla 2 Matriz de problemáticas de las estaciones Fuente: Elaboración propia

En esta fase se observó el comportamiento de la línea de panel en diferentes tiempos y con los diferentes productos (producto en proceso y producto terminado) esta información se presenta la tabla 3.

\begin{tabular}{|l|l|}
\hline \multicolumn{1}{|c|}{ Cliente Código } \\
\hline Allen puebla & J8t131a \\
\hline Cajas mil usos & H1325585 \\
\hline Clorox industrias & J8t177b \\
\hline $\begin{array}{l}\text { Crown } \\
\text { montacargas }\end{array}$ & H132606 \\
\hline Diper & J8s053a \\
\hline Jhon (epura) & J8y524 \\
\hline Embba & H1429656a \\
\hline Esther ivonne & H1430140-psa \\
\hline Ethan allen & J8y109 \\
\hline Fortipack & H0903546-gf \\
\hline Guardian & J8x020a \\
\hline Inomodul (diana) &
\end{tabular}

Tabla 3 Producto en proceso

Fuente: Elaboración propia

Antes de la implementación del sistema se deben tener algunos puntos en consideración: a) Contar con lugares visibles para operadores, b) Brindar suficiente información al personal sobre esta herramienta, de tal manera que estén pendientes sobre el proceso y c) Contar con trabajadores que tengan autonomía y criterio para activar las alarmas. El Sistema ANDON es una herramienta fácil para ejecutarse en cada una de las estaciones de trabajo que integran la línea de panel, sin embargo, solo se llevara a cabo en los puntos que sean considerados como críticos.

Durante las primeras semanas de observación se detectaron distintos problemas mencionados en la tabla 4.

\begin{tabular}{|c|c|c|}
\hline Código & $\begin{array}{c}\text { Defectos en línea de } \\
\text { panel }\end{array}$ & Descripción \\
\hline 302 & Hojas Delaminadas & $\begin{array}{l}\text { Pegamento } \\
\text { insuficiente }\end{array}$ \\
\hline 303 & Mal corte & Falta afilar cuchillas \\
\hline 305 & $\begin{array}{l}\text { Daños } \\
\text { montacargas }\end{array}$ & $\begin{array}{l}\text { Mal uso de } \\
\text { montacargas }\end{array}$ \\
\hline 306 & Falla en la máquina & $\begin{array}{ll}\text { Falta } & \mathrm{de} \\
\text { mantenimiento } & \\
\end{array}$ \\
\hline 307 & $\begin{array}{l}\text { Rompimientos de } \\
\text { core }\end{array}$ & $\begin{array}{l}\text { CHS no cumple con } \\
\text { calidad }\end{array}$ \\
\hline 308 & $\begin{array}{l}\text { Rompimiento de } \\
\text { papel }\end{array}$ & Demasiada tensión \\
\hline 316 & Velocidad & Retrasos \\
\hline 317 & $\begin{array}{l}\text { Problemas } \\
\text { Michelman }\end{array}$ & $\begin{array}{l}\text { Falta o derrame de } \\
\text { pintura }\end{array}$ \\
\hline 318 & $\begin{array}{l}\text { Piezas defectuosas } \\
\text { (Stacker) }\end{array}$ & $\begin{array}{ll}\text { Falta } & \mathrm{de} \\
\text { comunicación }\end{array}$ \\
\hline
\end{tabular}

Tabla 4 Tipo de defecto

Fuente: Elaboración propia 


\section{Análisis e identificación de problemas}

Durante esta fase de análisis se puede mostrar que hay fallas repetitivas ocasionando desperdicios debido al tiempo que transcurre para la reparación de la misma. En la tabla 5 se presenta un resumen cuantitativo de las fallas presentadas para hacer notar las posibles áreas de oportunidad.

\begin{tabular}{|r|l|r|}
\hline \multicolumn{1}{|c|}{ Código } & \multicolumn{1}{c}{ Defectos } \\
\hline 302 & Hojas Delaminadas & 212 \\
\hline 303 & Mal Corte & 200 \\
\hline 305 & Daños Por Montacargas & 88 \\
\hline 306 & Problemas De La Máquina & 88 \\
\hline 307 & Rompimientos De Core & 184 \\
\hline 308 & Rompimiento De Papel & 192 \\
\hline 316 & Tarimas Por Hora - Velocidad & 174 \\
\hline 317 & Problemas Con Michelman & 194 \\
\hline 318 & Piezas Defectuosas (Stacker) & 216 \\
\hline Defectos totales & 1548 \\
\hline
\end{tabular}

Tabla 5 Resumen de defectos en línea de panel Fuente: Elaboración propia

En el grafico 1 se clarifica la información referida en la tabla 3, a través de un Diagrama de Pareto, herramienta de calidad para identificar los defectos más frecuentes, las causas más comunes de los defectos o las causas más frecuentes de quejas de los clientes, este diagrama puede ayudar a concentrar los esfuerzos de mejoramiento en aquellas áreas en las que se puedan obtener las mayores ganancias.

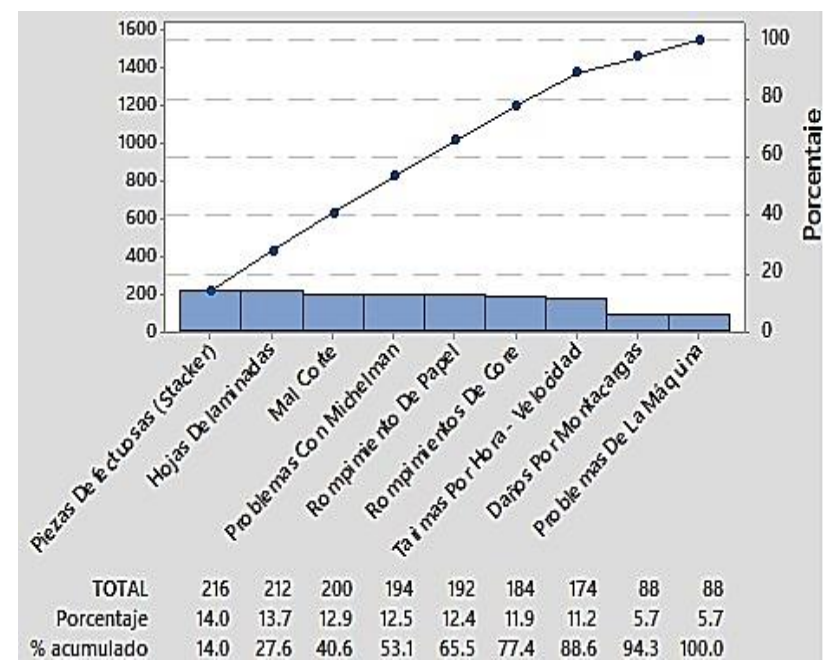

Gráfico 1 Resumen de defectos en línea de panel Fuente: Elaboración propia

El objetivo de este diagrama es ayudar a priorizar y a señalar la importancia de cada una de las áreas de oportunidad. De esta manera atacar los problemas con mayor impacto en la línea.
A través del diagrama de Pareto, se puede concluir que el $20 \%$ de los defectos, generan el $80 \%$ de las problemáticas, a través de este análisis se destacan 6 de 9 defectos críticos: Piezas defectuosas (Stacker), Hojas Delaminadas (Glue Coater), Mal corte (Trituradoras), Problemas con Michelman, Rompimiento de papel (Porta rollos) y Rompimiento de core (Mesa de expansión).

Cada uno de los paros que se presentan por estos defectos genera un consumo de todo tipo de insumos, ocasionando costos innecesarios los cuales elevan y encarecen el producto final. Como parte de este análisis se obtuvo el tiempo promedio que tarda el personal en atender los diferentes defectos y determinar el tiempo muerto total como se muestra en la tabla 6.

\begin{tabular}{|c|c|c|c|c|c|c|}
\hline Código & 302 & 303 & 307 & 308 & 317 & 318 \\
\hline $\begin{array}{l}\text { Tiempo } \\
\text { promedio } \\
\text { en } \\
\text { minutos }\end{array}$ & 5 & 5.2 & 6.7 & 10.2 & 11.9 & 3.1 \\
\hline $\begin{array}{l}\text { Tiempo } \\
\text { muerto } \\
\text { (minutos) }\end{array}$ & 1060 & 1040 & 1232.8 & 1958.4 & 2308.6 & 669.6 \\
\hline $\begin{array}{l}\text { Tiempo } \\
\text { total en } \\
\text { minutos }\end{array}$ & & & & & & 269.40 \\
\hline $\begin{array}{l}\text { Tiempo } \\
\text { en Horas }\end{array}$ & & & & & & 137.82 \\
\hline
\end{tabular}

Tabla 6 Total de tiempo muerto por cada tipo de defecto Fuente: elaboración propia

Se obtuvo un tiempo total de paro de 8269.4 minutos, es decir 137.82 horas. Con un promedio mensual de 4134.7 minutos, el tiempo obtenido es bastante elevado, considerando los gastos económicos relacionados con este tiempo por ejemplo los gastos por servicios de agua, energía eléctrica, combustibles, esta información se presenta en la tabla 7.

\begin{tabular}{|l|r|}
\hline Costo Promedio mensual por consumo de energía & (USD) \\
\hline Agua & 3780.00 \\
\hline Energía Eléctrica & 29498.00 \\
\hline Gas & 7140.00 \\
\hline Total & 40418 \\
\hline Costo diario & 2020.9 \\
\hline Costo / Hora & 168.40 \\
\hline
\end{tabular}

Tabla 7 Costo de insumos y servicios Fuente: Elaboración propia 
En la Tabla 7 se puede observar el costo mensual de energía consumido durante un periodo de tres meses, para obtener un porcentaje aproximado de tiempo muerto sin tomar en cuenta la mano de obra se utilizarán los datos de producción plasmados en la tabla 7 , estos datos nos proporcionarán el porcentaje de tiempo muerto durante los tres meses.

Tiempo disponible $=3$ meses $* 20$ días $*$ 12 horas $* 60$ minutos $=28800$ minutos.

$\%$ de tiempo de paros $=(8269.4$ minutos $/ 28800$ minutos) $* 100=28.71 \%$.

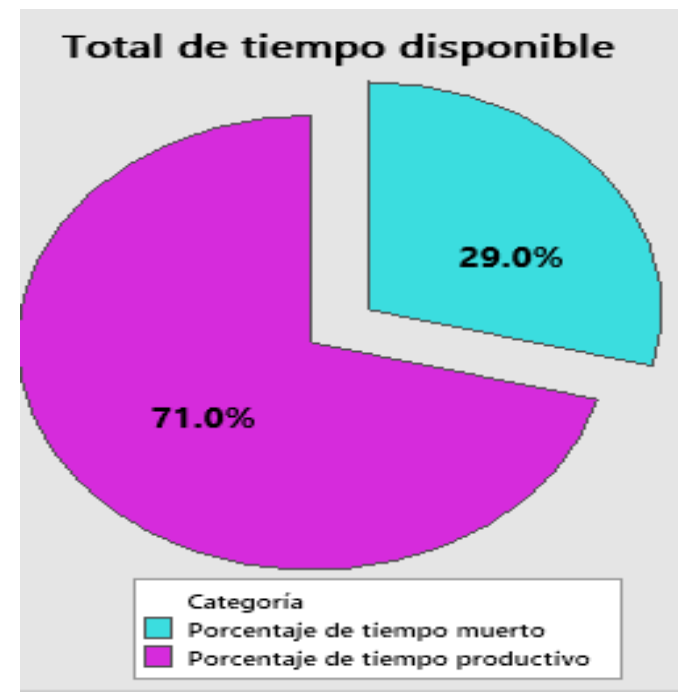

Gráfico 2 Porcentaje tiempo disponible en línea de panel Fuente: elaboración propia

En el gráfico 2, se muestra el porcentaje real de tiempo productivo en la línea de panel, con este importante hallazgo resalta la necesidad de la implementación del sistema ANDON como una herramienta que revolucione y optimice la línea de panel a través de su correcta aplicación.

\section{Implementación del Sistema ANDON}

Para esta fase se describirá como fue atendido el problema de acuerdo con el sistema ANDON y que elementos fueron implementados para la reducción o eliminación del error.

En el área de Stacker la falla que se generaba con más frecuencia es el código 318 (Piezas defectuosas) este proceso se ubica al final de la línea, en donde el operador se encarga de estibar las placas de cartón en tarimas, siendo esta sub-área la que presenta el error con mayor puntuación.
En la estación de trabajo fue instalado un dispositivo para el control visual (interruptor de botón momentáneo) figura 4 , esta proporciona una señal de aviso en situaciones urgente y puede utilizarse con diferentes sonidos según sea su aplicación, se basa principalmente en la implementación de un botón pulsador en con junto de una ayuda auditiva.

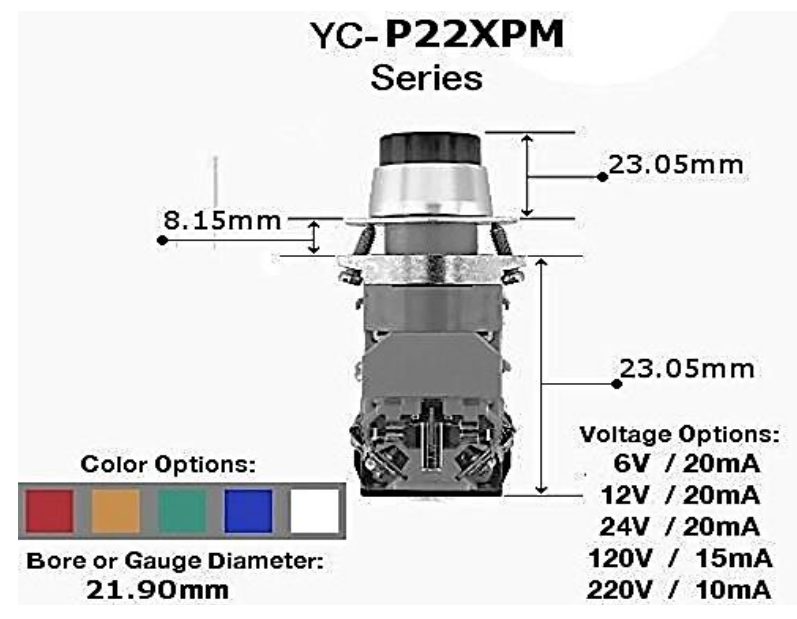

Figura 4 Interruptor de botón momentáneo

Fuente: Recuperado de https://www.google.com/search?

Al implementar este dispositivo se obtendrán beneficios como la disminución de tiempo muerto, disminución de scrap y un cuantioso ahorro económico. Esta alarma tendrá como principal función dar aviso al líder de la línea para la corrección inmediata del problema, en la figura 5 se muestra la instalación del dispositivo.

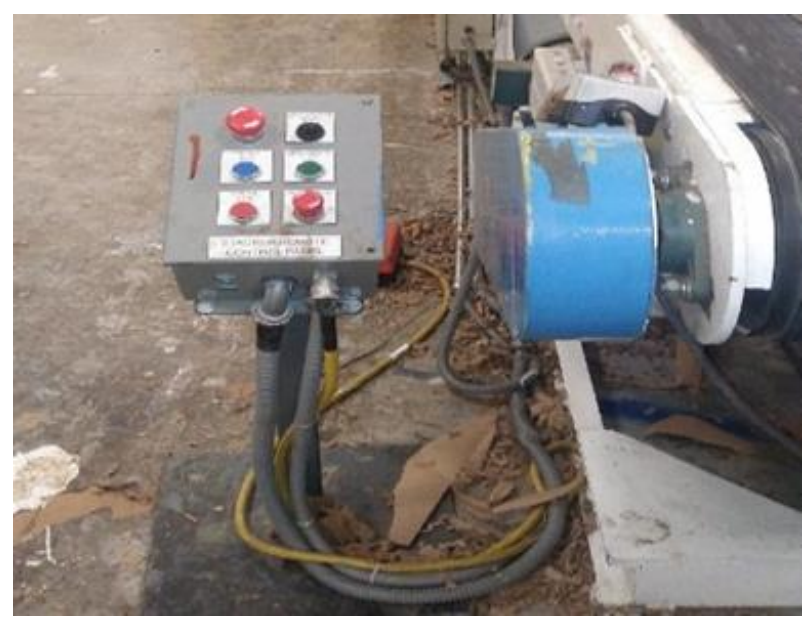

Figura 5 Instalación de Interruptor de botón momentáneo Fuente: Empresa (caso de estudio) 
En la estación de trabajo Glue Coater el código 302 (hojas delaminadas) se presenta como un problema crítico, se procede a instalar un sensor y una electro válvula, la principal función es indicar los niveles de pegamento así como el encender una alerta cuando está al 15\% de su capacidad, esta implementación tuvo una desempeño favorable al aumentar la disponibilidad de este proceso manteniendo un flujo de trabajo constante y aumentando su productividad, en la figura 6 se muestran los componentes del interruptor de flotador en acero inoxidable con sensor de nivel e imágenes de su instalación en la estación de trabajo mencionada.

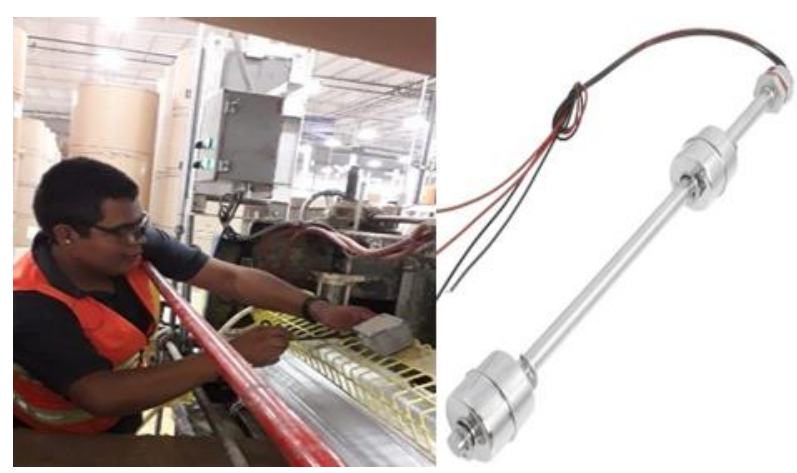

Figura 6 Instalación de Interruptor de botón momentáneo Fuente: Empresa (caso de estudio)

El proceso de triturado tiene como objetivo eliminar la rebaba de papel que se obtiene al sacar los diferentes productos en esta estación, la falla detectada es la 303 (problemas en el corte), se implementó un sensor interruptor de límite con la capacidad de detectar rebaba sobrante, evitando que pase al siguiente proceso y alertando con ayuda auditiva al operador para su inmediata solución, en la figura 7 se muestra la instalación del sensor en la estación de trabajo.

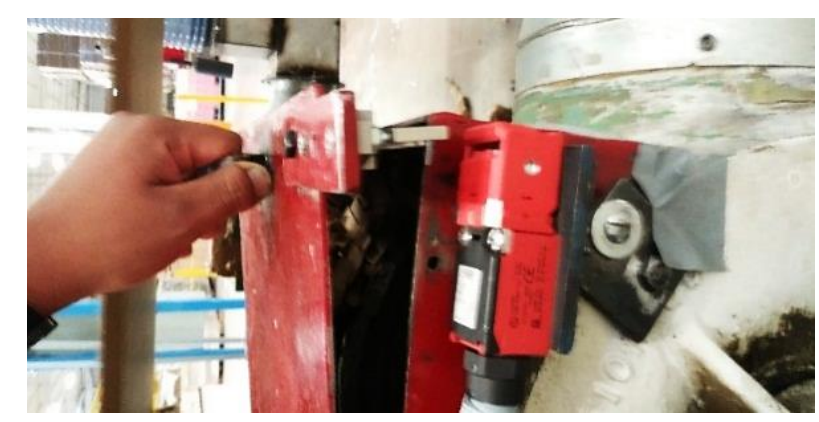

Figura 7 Instalación de sensor Interruptor de limite Fuente: Empresa (caso de estudio)
El siguiente código atendido, es el 317 (falta o derrame de recubrimiento) este se origina en el área de Michelman, esta área se encarga de aplicar un recubrimiento de color rojo solo para material que va destinada a un cliente en específico, anteriormente se tenía que vigilar de manera manual el depósito donde se coloca el recubrimiento, de no hacerlo así, la pintura se derrama y como consecuencia contrae pérdida económica, tiempo muerto para llevar a cabo la limpieza y retraso en el pedido del cliente.

Por otra parte, el material que sale sin el recubrimiento se tira, puesto que no es aceptado por el cliente, también si el operador demora en rellenar el depósito el papel se pega en los tubos, ocasionando el rompimiento de este teniendo como consecuencia mayor cantidad de scrap, y un paro total de la línea.

Para la solución de este código se contempló la instalación de una alarma, un sensor de proximidad y un electroválvula, como se muestra en la figura 8 , con el propósito de alertar cuando esté a punto de ser llenado el recipiente y este cierre de manera autónoma, así como el poder visualizar los niveles del depósito y controlar el suministro del recubrimiento de manera automática sin causar ninguna afectación al proceso y al producto.

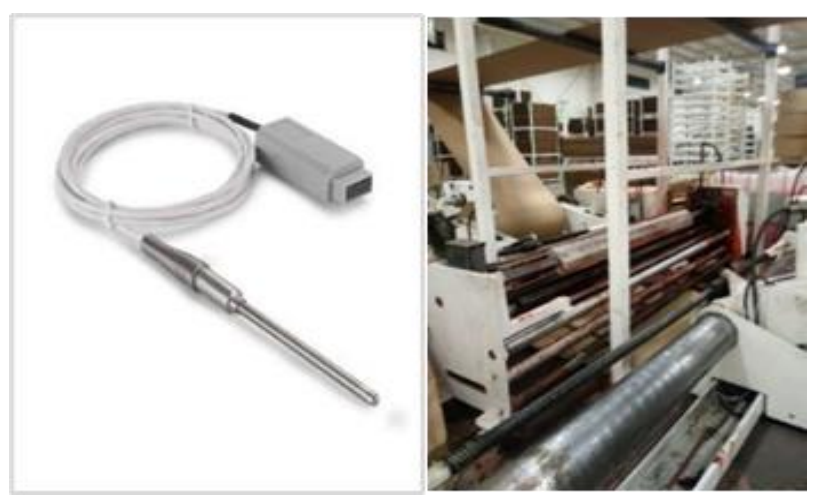

Figura 8 Instalación de sensor interruptor flotador de acero inoxidable y sensor de nivel de líquido Fuente: Empresa (caso de estudio)

Algunos de los beneficios con este dispositivo ANDON, es la reducción en el gasto innecesario del recubrimiento, no habrá tiempo perdido limpiando el área porque no se volverá a tirar la pintura, la cantidad de scrap disminuirá considerablemente, el tiempo de arranque será más rápido. 
En la tabla 8 se muestra un formato realizado e implementado en la línea de panel, con la finalidad de obtener información en tiempo real para la mejora constante del proceso y poder continuar detectando áreas de oportunidad.

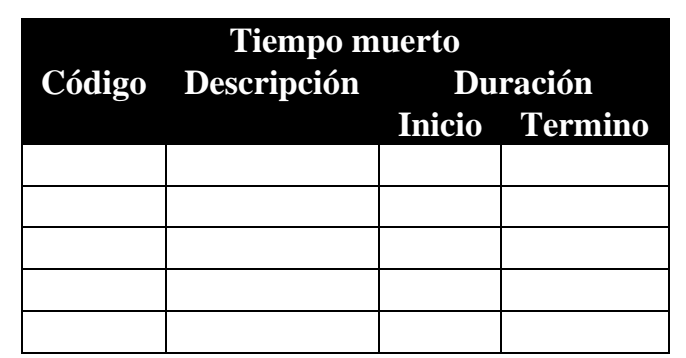

Tabla 8 Check List (registro de fallas en línea de panel) Fuente: elaboración propia

En el área de Stacker se implementó una tabla como ayuda visual con el objetivo de mostrar al operador como debe realizar una inspección del material, para identificar si el material cumple con las especificaciones solicitadas, esta información se muestra en la figura 9 está integrada por imágenes y texto concreto.

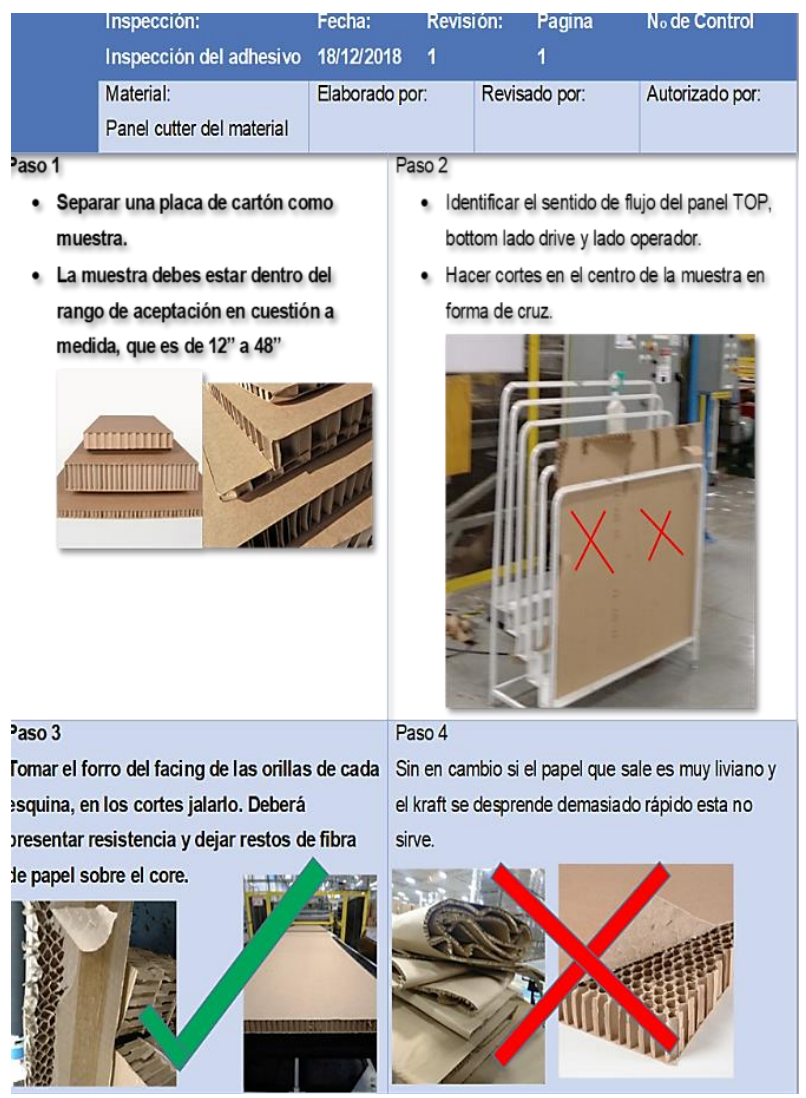

Figura 9 Ayuda visual (área de Stacker)

Fuente: Empresa (caso de estudio)
A continuación, se muestran en la tabla 9, los costos por concepto de implementación del sistema ANDON de acuerdo con todos los dispositivos instalados en las distintas áreas que integran la línea de panel.

\begin{tabular}{|c|c|}
\hline \multicolumn{2}{|c|}{ Costo por implementación del sistema ANDON } \\
\hline Concepto & Costo en USD \\
\hline Stacker & $\$ 808.00$ \\
\hline Glue Coater & $\$ 205.00$ \\
\hline Trituradoras & $\$ 245.00$ \\
\hline Michelman & $\$ 230.00$ \\
\hline Porta rollos & $\$ 1049.4$ \\
\hline Mesa de expansión & $\$ 305.00$ \\
\hline Mano de obra & $\$ 255.00$ \\
\hline Servicios & $\$ 4644.47$ \\
\hline Horas extras & $\$ 2482.2$ \\
\hline Total & $\$ 10,224.07$ \\
\hline
\end{tabular}

Tabla 9 Inversión por implementación del sistema ANDON en línea de Panel

Fuente: elaboración propia

\section{Resultados}

En este apartado se describen los hallazgos obtenidos a través de la implementación del sistema ANDON, en el gráfico 3 se muestra un comparativo relacionado con la producción de tarimas en la línea de panel (producto terminado), así mismo se obtuvo una reducción del $58.82 \%$ en tarimas rechazadas incrementando la estabilidad del proceso, por otra parte, la producción se incrementó en 1581 tarimas, generando mayores ganancias para la empresa.

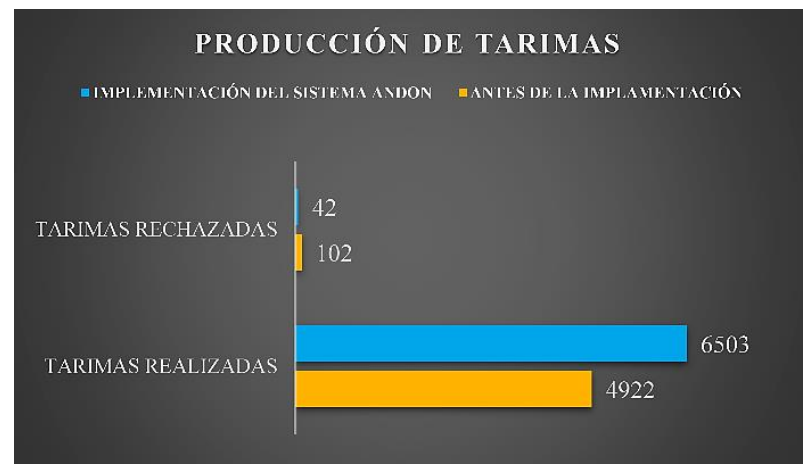

Gráfico 3 Porcentaje tiempo disponible en línea de panel Fuente: Elaboración propia 
En el gráfico 4 se muestra una comparación de acuerdo con el tipo de falla presentada antes y después de implementación del sistema ANDO, los hallazgos son favorables, puesto que se presentó una reducción sistemática en las fallas, esto beneficia el flujo operativo de las estaciones que integran la línea de panel.

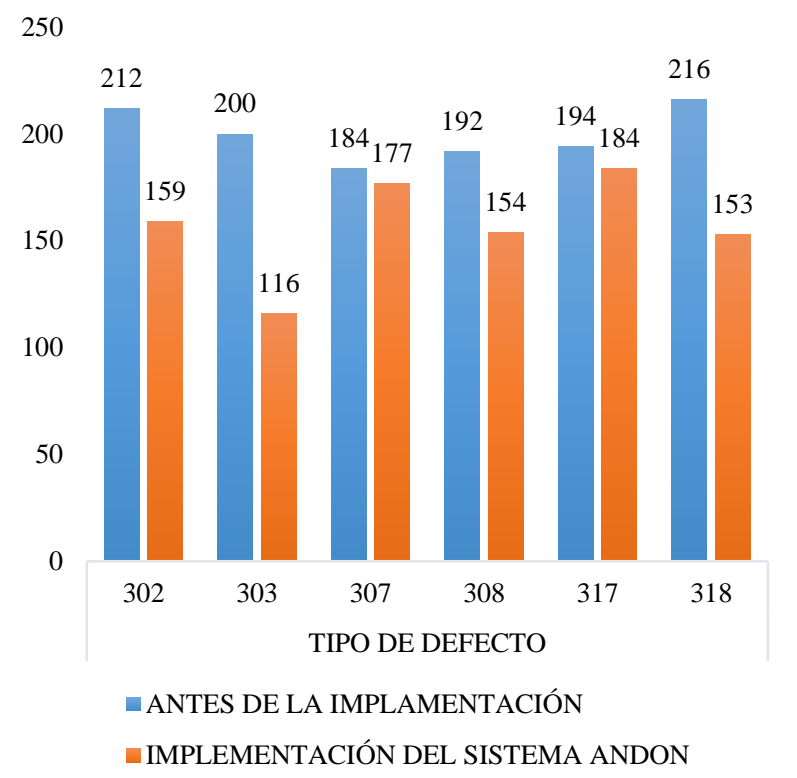

Gráfico 4 Comparativa tipo de defecto Fuente: Elaboración propia

Antes de la implementación del sistema ANDON se tenía un promedio de 199 defectos como se muestra en el gráfico 5, el intervalo de confianza del $95 \%$ para la media es de 184 a 216 defectos.

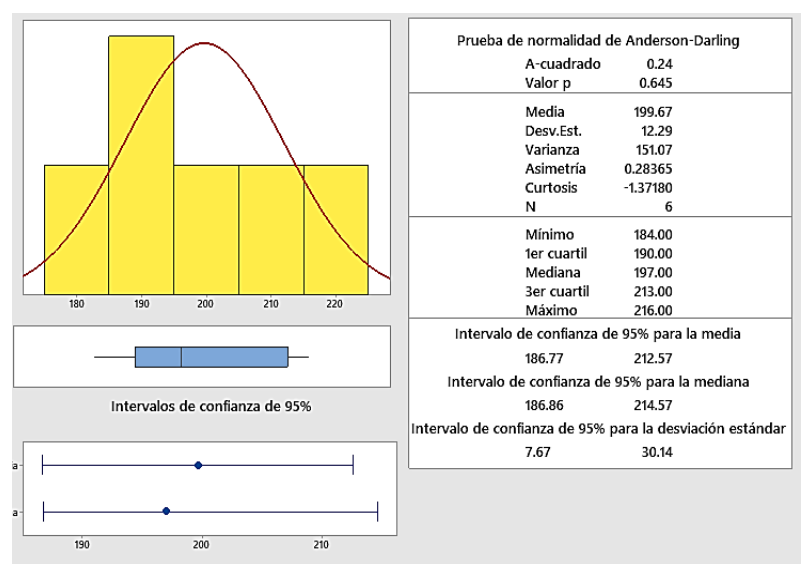

Gráfico 5 Resumen gráfico de los diferentes defectos antes de la implementación Fuente: Elaboración propia
En el gráfico 6 se muestra la media de defectos después de la implementación, teniendo una reducción en la media de defectos con un valor de 157, el intervalo de confianza del $95 \%$ para la media es de 132 a 182 defectos, con esta herramienta estadística se comprueba nuevamente la efectividad del sistema implementado en la línea de panel.

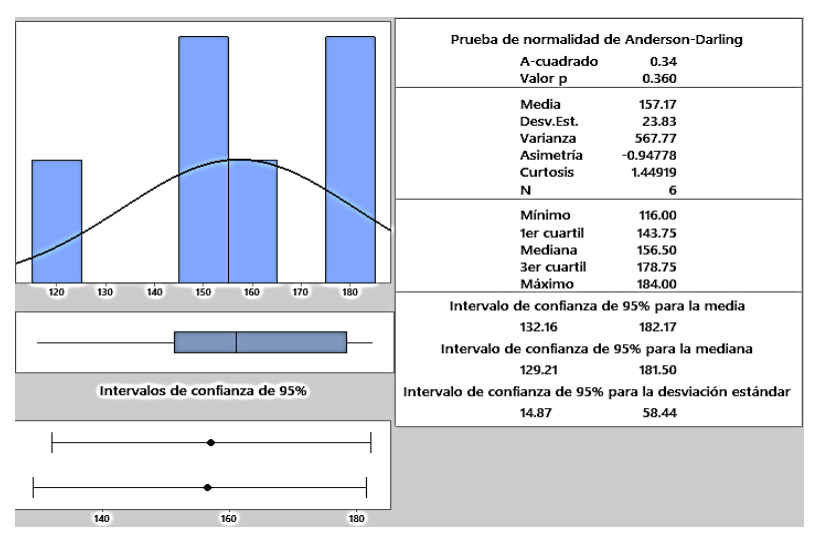

Gráfico 6 Resumen gráfico de los diferentes defectos posterior a la implementación Fuente: Elaboración propia

El gráfico 7 presenta los tiempos de respuesta en minutos por cada tipo de defecto antes y posterior a la implementación del sistema ANDON, cabe señalar que este es uno de los objetivos principales y que fue logrado de acuerdo con la evidencia presentada.

\section{— ANTES DE LA IMPLAMENTACIÓN \\ - IMPLEMENTACIÓN DEL SISTEMA ANDON}

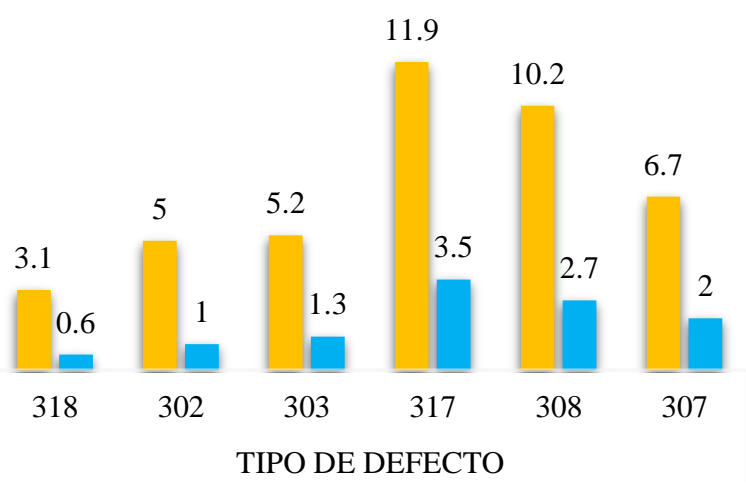

Gráfico 7 Comparativo del tiempo de respuesta (minutos) Fuente: Elaboración propia

Después de la implementación del sistema ANDON se determinó el tiempo muerto total como se muestra en la tabla 10 , se eliminó un total de 110.24 horas que no agregan valor al proceso, por el contrario, fue convertido en actividades productivas e impactando de manera favorable en la línea de panel. 


\begin{tabular}{|l|r|r|r|r|r|r|}
\hline \multicolumn{1}{|c|}{ Código } & $\mathbf{3 0 2}$ & $\mathbf{3 0 3}$ & $\mathbf{3 0 7}$ & $\mathbf{3 0 8}$ & $\mathbf{3 1 7}$ & $\mathbf{3 1 8}$ \\
\hline $\begin{array}{l}\text { Tiempo } \\
\text { promedio } \\
\text { en } \\
\text { minutos }\end{array}$ & 1 & 1.3 & 2 & 2.7 & 3.5 & 0.6 \\
\hline $\begin{array}{l}\text { Tiempo } \\
\text { muerto } \\
\text { (minutos) }\end{array}$ & 159 & 150.8 & 294 & 415.8 & 549.5 & 85.8 \\
\hline $\begin{array}{l}\text { Tiempo } \\
\text { total en } \\
\text { minutos }\end{array}$ & \multicolumn{10}{|c|}{1654.9} \\
\hline $\begin{array}{l}\text { Tiempo } \\
\text { en Horas }\end{array}$ & \multicolumn{10}{|c|}{27.58} \\
\hline
\end{tabular}

Tabla 10 Total de tiempo muerto con la implementación del sistema ANDON

Fuente: Elaboración propia

Al hablar de productividad nos referimos a la mejora continua del proceso, ya que no solo se busca una producción rápida, también buscamos producir mejor.

De acuerdo con los valores obtenidos se entiende que el aumento de la productividad en la empresa antes y después de la implementación del sistema ANDON, en relación con la producción hubo un aumento del $32.12 \%$ (1581 tarimas), considerando que el costo promedio por tarima es de $\$ 28.03$ dólares se obtuvo una ganancia de $\$ 44,315.43$ dólares.

Con la implementación de este proyecto se generó un ahorro por concepto de servicios (agua, energía eléctrica, combustibles), tal y como se muestra en el gráfico 8, cabe señalar que con la implementación del sistema se evitó un gasto de $\$ 18,564.41$ dólares mensuales para la empresa.

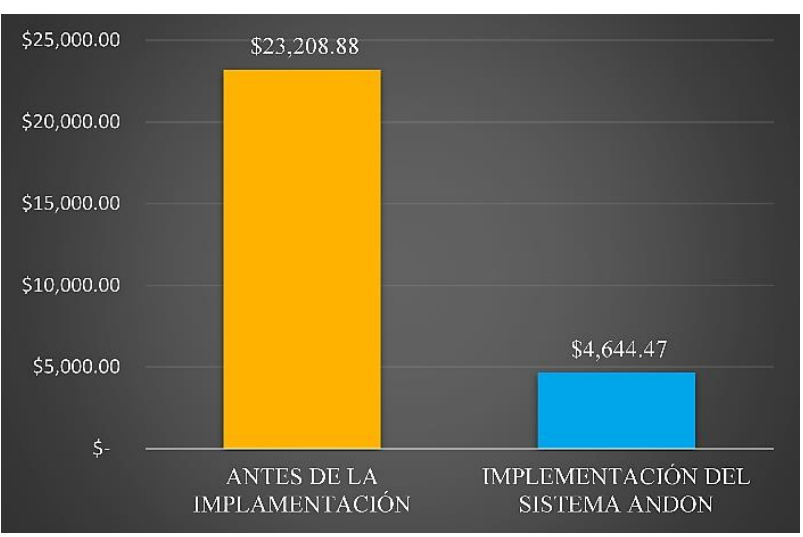

Gráfico 8 Comparativo del gasto en servicios antes y después de la implementación

Fuente: Elaboración propia
Otro beneficio fue la reducción de horas extraordinarias, anteriormente esta era una práctica recurrente para poder cumplir con las metas de producción diarias, en el gráfico 9 se muestra el comparativo en términos económicos antes y después de la implementación.

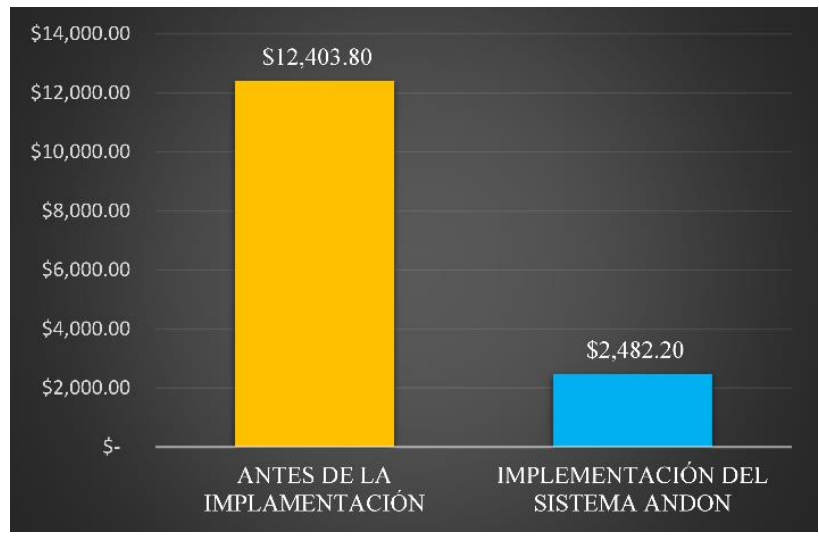

Gráfico 9 Comparativo horas extra antes y después de la implementación

Fuente: elaboración propia

\section{Agradecimiento}

A la carrera de Ingeniería Industrial del Instituto Tecnológico Superior de Huauchinango por el apoyo y las facilidades en el desarrollo de este proyecto, así mismo, agradezco el trabajo colegiado de los miembros del Cuerpo Académico Tecnología Aplicada con número de registro ITESHUAU-CA-2.

\section{Conclusiones}

Actualmente las empresas están obligadas a analizar sus tiempos muertos relacionados con su operación diaria, ocasionan el consumo extraordinario de todo tipo de insumos, también se generan actividades que no agregan valor al proceso y encarecen el costo del producto final.

Generalmente los tiempos muertos o de espera se presentan cuando ocurre una falla en la línea de producción y no es corregida en su momento. La automatización industrial es una disciplina que involucra principalmente a las especialidades de la ingeniería electrónica, industrial, mecánica, mecatrónica y de sistemas computacionales que va desde un sencillo "sistema de control"; hasta la instrumentación industrial, sistemas de control y supervisión, los sistemas de transmisión y recolección de datos y las aplicaciones de software en tiempo real para supervisar y controlar las operaciones de procesos y plantas industriales (Poma, 2011). 
En este caso de estudio se actualizó y revoluciono el sistema de producción de la línea de panel con la implementación del sistema ANDON, mismo que contiene diversos dispositivos instalados en las estaciones de trabajo que integran esta línea.

Además, se logró una comunicación más rápida para la solución de contingencias en las estaciones de trabajo, por otra parte, el sistema implementado permite el registro de cada uno de los tipos de defectos, así como el tiempo de paro y la causa de la problemática, la información registrada puede ser examinada por el supervisor de la línea en tiempo real, contribuyendo a la toma de decisiones, mejorando el flujo del proceso.

De acuerdo con la implementación del sistema ANDON se consiguieron los siguientes beneficios:

- Después de las pruebas que se realizaron en la línea de panel y una vez implementada la herramienta, se concluye que se cumplieron los objetivos planteados.

Se eliminó un total de 110.24 horas que no agregan valor al proceso, también se incrementaron de manera favorable $y$ sistemática los tiempos de respuesta.

- Se mejoró el flujo de comunicación en la línea de panel, generando confianza y trabajo colaborativo entre los operadores y supervisores.

También se logró una reducción del $58.82 \%$ en tarimas rechazadas, dando como resultado un proceso más confiable y de calidad.

- $\quad$ La producción aumento en 32.12 \% (1581 tarimas), es decir, una ganancia de $\$ 44,315.43$ dólares.

Se deberá realizar una constante motivación del equipo de trabajo para continuar usando el sistema ANDON, realizando reportes de los nuevos problemas, recalcando que el personal de mantenimiento deberá estar al tanto de las señales de este sistema, es parte fundamental para la solución del problema.
Para que esta herramienta funcione correctamente, el operador debe ser responsable y cuidadoso de utilizarlo correctamente, activando las alarmas solo para verdaderas emergencias.

Profundizar el estudio de los nuevos modelos de producción como la manufactura esbelta y su incidencia en los procesos de las industrias, de igual manera conceptos como el mantenimiento productivo total (TPM).

\section{Referencias}

Ávila, B. L. (11 de diciembre de 2015). Emprendices. recuperado de https://www.emprendices.com

Arango, M. J. (2013). Aplicación de herramientas de manufactura esbelta para el mejoramiento de la cadena de valor de una línea de producción de sillas para oficina.

Cruz, I. B., \& Aguilar, J. A. (2020). La gestión LEAN del tiempo: Método LTM para ser más ágil y efectivo trabajando menos y mejor. Editorial UOC.

Gutiérrez Pulido H. (2010). Calidad total y productividad, Tercera edición, México, D.F., McGraw-Hill.

Esquivel V. J. (2009). Metodología para la detección y prevención de Fallas en equipos industriales de producción [Tesis de Maestría, Universidad Nacional Autónoma de México] http://www.ptolomeo.unam.mx:8080/jspui/bitst ream/132.248.52.100/2872/1/esquivelvillar.pdf

Montalvo, W., Llamuca, E. S., Benalcazar, F. G., Garcia, C. A., \& Garcia, M. V. (2020). Sistemas de automatización de bajo costo para la integración industrial de plantas basadas en AMQP. Revista Ibérica de Sistemas e Tecnologias de Informação, (E27), 121-134.

Poma D. J., Tello Y. R., Berrospi Q. E., (2011). Gestión de base de datos con SCADA para control automatizado de una válvula de control proporcional. Sistema e informática, Revista de la Facultad de Ingeniería Industrial, Universidad Nacional Mayor de San Marcos, Perú 
Roqueme Salazar. E., Suarez Ballesteros L. (2015). Implementación de la Metodología Lean para el mejoramiento del proceso comercial de la pyme Tres60 logística, Bogotá DC, Universidad Militar Nueva Granada.

Saá Tapia, F. D., Noboa Castro, A. J. (2018). Implementación del sistema ANDON en el área de aparado central para la gestión automática de imprevistos en un proceso productivo de la empresa plasticaucho industrial [Tesis de Licenciatura, Universidad Tecnológica Indoamérica]http://repositorio.uti.edu.ec//handl e/123456789/1059

Socconini L. (2008). Lean Manufacturing: Paso a paso, México; Editorial Norma Ediciones.

Vera Torres R., Alvarado Santander L. (2018). Industria 4.0, Prendas Inteligentes: Andon como Sistema de Información para la toma de decisiones. Revista Aristas: Investigación Básica y Aplicada, 6(12), 116-121.

Pérez Vergara, I. G., \& Rojas López, J. A. (2019). Lean, Seis Sigma y Herramientas Cuantitativas: Una Experiencia Real en el Mejoramiento Productivo de Procesos de la Industria Gráfica en Colombia // Lean, Six Sigma and Quantitative Tools: A Real Experience in the Productive Improvement of Processes of th. Revista De Métodos Cuantitativos Para La Economía Y La Empresa, 27, 259-284. Recuperado a partir de https://www.upo.es/revistas/index.php/RevMet Cuant/article/view/3218

Wilches Arango M., Cabarcas Reyes, J. (2013). Aplicación de herramientas de manufactura esbelta para el mejoramiento de la cadena de valor de una línea de producción de sillas para oficina. Dimensión empresarial, 11, 126-136. 